\title{
Depression - ein komplexes dynamisches System
}

\author{
Günter Schiepek
}

Psychotherapie-Wissenschaft 10 (2) 49-58 2020

www.psychotherapie-wissenschaft.info

CC BY-NC-ND

https://doi.org/10.30820/1664-9583-2020-2-49

\begin{abstract}
Zusammenfassung: Der vorliegende Beitrag befasst sich mit neueren Befunden zur Depressionsforschung, vor allem zur psychotherapeutischen Behandlung von Depression. Auf Wunsch der Redaktion sollte damit auch eine Synopse der wissenschaftlichen und praktischen Entwicklungen gegeben werden, die am Institut für Synergetik und Psychotherapieforschung der Paracelsus Medizinischen Privatuniversität Salzburg und der kooperierenden Forschergruppen stattfinden. Ein Schwerpunkt liegt in der Personalisierung der Psychotherapie und betrifft sowohl neuere Methoden der Fallkonzeption (z. B. idiografische Systemmodellierung und deren computerbasierte Visualisierung) als auch Verfahren des hochfrequenten Prozessmonitorings und Prozessfeedbacks. Hierfür wurde das Internet- und App-basierte Synergetische Navigationssystem (SNS) entwickelt und erprobt. Die Verläufe der Psychotherapie depressiver Patient*innen zeigen diskontinuierlich Sprünge dynamischer Muster (sogenannte Ordnungsübergänge), die von einer erhöhten kritischen Instabilität (dynamische Komplexität) und zeitlich begrenzter Synchronisation der Items eines Prozessbogens eingeleitet werden. Erhöhte kritische Instabilität ist zudem prädiktiv für besseren Therapieerfolg. In einer Studie an komorbid depressiven Patient*innen mit Zwangsstörungen wurde deutlich, dass sich neben klinischen Verbesserungen auch die funktionelle Konnektivitätsdynamik neuronaler Netze verändert. Wiederholte fMRT-Scans zeigten, dass Flexibilität und Fluidität der Neurodynamik mit der Psychopathologie (z. B. Depressionsintensität, BDI) negativ assoziiert sind, aber im Verlauf von Psychotherapie zunehmen. Abschliessend erfolgt eine Einschätzung von Möglichkeiten, nichtinvasive Neurostimulation mit Psychotherapie zu kombinieren.
\end{abstract}

Schlüsselwörter: personalisierte Psychotherapie, Prozessmonitoring, therapeutische Verlaufsmuster, Ordnungsübergänge, Frühwarnindikatoren, neuronale Prozessmuster, nichtinvasive Neurostimulation

\section{Problemstellung}

Depression ist ein gravierendes Public Health-Problem, mit einer erheblichen Prävalenz- und Inzidenzrate. Allein in Europa erhalten mehr als 10 Millionen Menschen pro Jahr eine entsprechende Diagnose (Major Depressive Disorder, MDD) (Whiteford et al., 2013). Depressionen führen zu einem starken subjektiven Leiden mit Symptomen wie Niedergeschlagenheit, Gefühlsarmut (numbness), Antriebsarmut und Motivationsverlust, Hoffnungslosigkeit, Schlafstörungen, Appetitlosigkeit und anderen, zu erhöhten Suizidraten, aber auch zu ökonomischen Folgeschäden im individuellen Bereich und in der Dimension ganzer Volkswirtschaften (Greenberg et al., 2015). Behandlungsmöglichkeiten existieren (vor allem Pharmakotherapie und Psychotherapie), sind aber in vielen Fällen nicht nachhaltig genug und können Rezidive nicht verhindern. 20-30\% aller Patient*innen sprechen auf Standardbehandlungen nicht an und gelten als behandlungsresistent (Olchanski et al., 2013). Somit kann die Entwicklung neuer Therapiemöglichkeiten als wichtig und dringlich gelten.

Ein Weg dieser Entwicklungen geht in Richtung störungsspezifischer Behandlungsmanuale, wobei vor allem kognitive Verhaltenstherapie in Einzel- und Gruppensettings als Teil internationaler Behandlungsrichtlinien gilt (Butler et al., 2006) und sich als ähnlich wirksam wie antidepressive Pharmakotherapie erwiesen hat (DeRubeis et al., 2008), allerdings auch nicht als (substanziell) wirksamer als andere bona fide und kompetent durchführte Psycho- therapieansätze (Shedler, 2018; Wampold et al., 2017). Der Dodo-Bird-Effekt, der in zahlreichen Meta-Analysen bestätigt und repliziert werden konnte ( «Unterschiedliche psychotherapeutische Ansätze haben im direkten Vergleich ähnliche Effekte»), schlägt offenbar auch im Bereich der Depressionstherapie zu (Wampold et al., 2018).

\section{Personalisierte Psychotherapie}

Ein anderer Weg geht in Richtung personalisierter Psychotherapie, was bedeutet, dass sich Interventionen nicht nur am Störungsbild orientieren, sondern auch am aktuellen Entwicklungsstand einer Therapie (Schiepek, 2019). Personalisierung erfordert ein prozesssensitives Vorgehen, das unter Nutzung entsprechender Entscheidungsheuristiken eine Auswahl von Methoden und Ansatzpunkten trifft. Eine Voraussetzung hierfür ist, die Veränderungsprozesse tatsächlich zu erfassen und auf der Höhe des Geschehens $\mathrm{zu}$ analysieren und zu visualisieren, was mit Internet- und App-basierten Methoden möglich ist. Patient*innen schätzen dabei den Stand ihrer Entwicklung, verschiedene Emotionen, ihre Veränderungsmotivation, die Therapiebeziehung, Mentalisierung, Körpererleben und andere prozessrelevante Aspekte regelmässig (z.B. täglich) ein und besprechen die resultierenden Prozessmuster in den Sitzungen mit ihrem Therapeuten bzw. ihrer Therapeutin (Abb. 1). Benutzt werden hierfür standardisierte Prozessfragebögen wie der Therapie-Prozessbogen (TPB, Schiepek et al., 2019) oder 


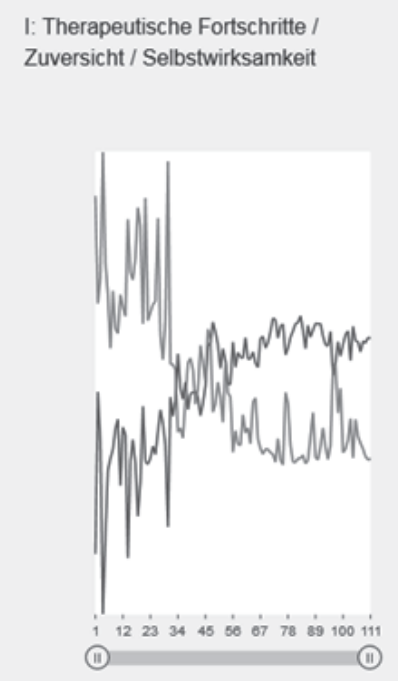

VIII: Selbstfürsorge / Körpererleben

V: Verănderungsmotivation

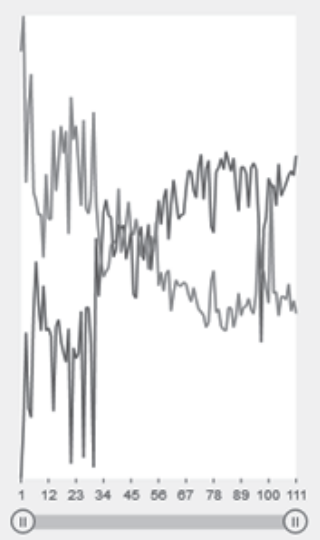

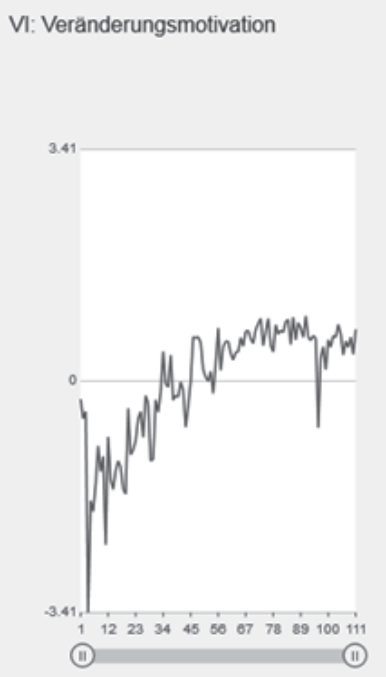

III: Beziehung und Vertrauen zu den Therapeut_innen

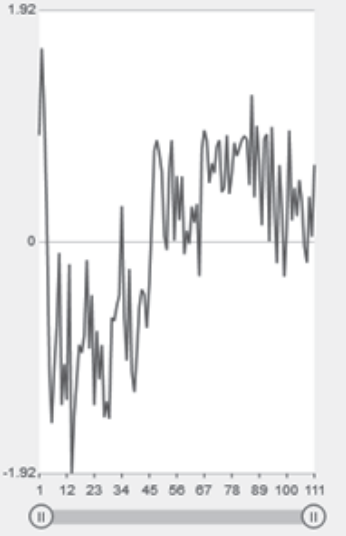

Abb. 1: Therapieprozess eines Patienten mit Depression und Zwangsstörung. Zeitreihen von links nach rechts: Therapeutische Fortschritte/Zuversicht/Selbstwirksamkeit (überlagert von Symptom- und Problembelastung), Selbstfürsorgel Körpererleben (überlagert von dysphorischer Emotionalität), Veränderungsmotivation, therapeutische Beziehung. $X$-Achse: Therapiedauer in Tagen. Erkennbar ist ein Musterwechsel (Ordnungsübergang) nach etwa einem Drittel der Therapie, mit starken kritischen Fluktuationen im zeitlichen Vorfeld.

aber personalisierte Fragebögen, die auf der Basis einer umfassenden Fallkonzeption gemeinsam mit den Patient"innen erstellt werden. Erkennbar ist in jedem Fall, dass Therapieprozesse keinem Standardverlauf folgen, sondern hoch komplex und individuell verlaufen (Schiepek et al., 2020).

Ein Vorgehen, das sich für kooperative Fallkonzeptionen in besonderer Weise eignet, ist die idiografische Systemmodellierung, bei der die relevanten Aspekte des Problemgeschehens und der Art der Verarbeitung durch Patient*innen (Symptome, Beschwerden, aber auch Ressourcen, Kompetenzen, Bewertungen, Copingstrategien etc.) als Variablen herausgearbeitet und ihre Wechselwirkungen in Form von Pfeilen visualisiert werden. Ein solches Modell liefert eine multi-perspektivische, systemische und

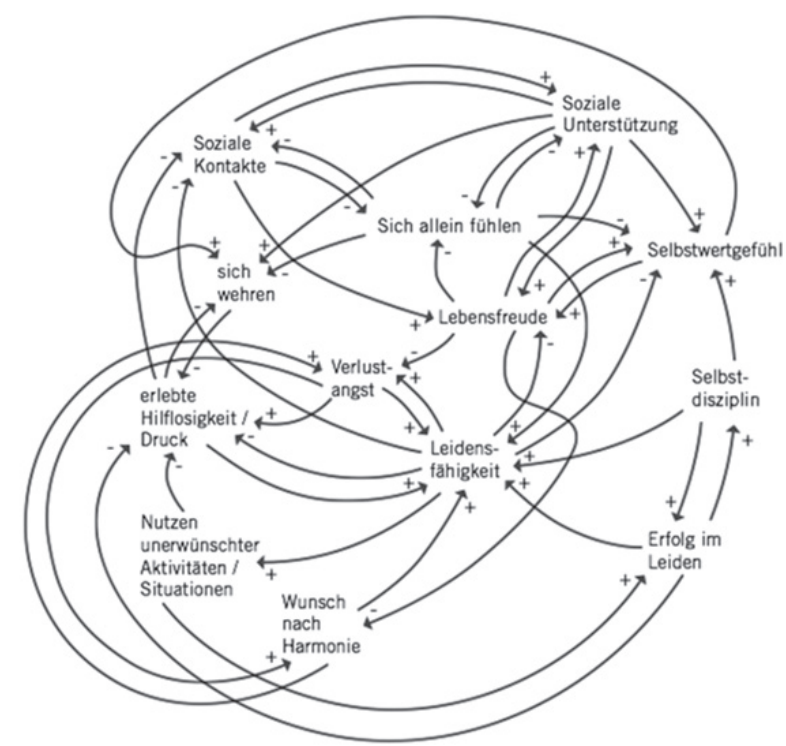

Abb. 2: Beispiel eines idiografischen Systemmodells einer Patientin mit depressiver Belastung dynamische Betrachtung des Problemszenarios, aber auch möglicher Ansatzpunkte für Veränderung und persönliche Entwicklung (Abb. 2). Die Variablen des Modells können in Fragen zur Selbsteinschätzung übersetzt werden und sind dann über Internet oder App zur Beantwortung verfügbar. Die Fragebogenbeantwortung sowie die Analyse und Visualisierung erfolgt mithilfe des Synergetischen Navigationssystems (SNS, Schiepek et al., 2018a, b).

Beispiele für Systemmodelle und Prozesserfassungen liegen für Patient*innen mit der Hauptdiagnose Depression oder aber mit depressiver Belastung bei anderen Hauptdiagnosen vor (Kronberger \& Aichhorn, 2015; Schiepek et al., 2013a; 2016, 2018b). Die Effekte eines solchen personalisierten Vorgehens sind vielfältig: Bereits die Modellierung in Kooperation mit Patient*innen eröffnet neue und vielfältige Perspektiven auf das Problem und macht systemische Zusammenhänge zwischen unterschiedlichen Aspekten deutlich, womit eine kognitiv-emotionale Umstrukturierung angeregt wird. Ziele und Interventionen beziehen sich nicht (nur) auf «die Depression», sondern auf Variablen und Relationen, die im Systemmodell dargestellt sind, womit sie erheblich spezifischer werden. Die Prozesse und Dynamiken der Variablen werden erfasst und dargestellt, was die Grundlage für datenbasierte Fallreflexionen mit Patient*innen liefert. Tägliche Selbsteinschätzungen während der Therapie und darüber hinaus, zum Beispiel bei Settingwechseln oder während eines Nachbehandlungszeitraums, fokussieren auf Themen, Inhalte, Therapieziele und Ressourcen im Alltag, die ansonsten weniger im Fokus stünden (öko-systemische Perspektive). Therapeut*in und Patient" in erhalten Hinweise auf sensitive Phasen im Prozess, die sinnvoll genutzt werden können. Entscheidungsheuristiken wie die generischen Prinzipien (Haken \& Schiepek, 2006; Rufer, 2012) liefern Hinweise auf geeignete nächste Schritte im Prozess. 
Im Rahmen eines personalisierten Prozesses können Interventionen system- und prozessspezifisch eingesetzt werden, und bereits die Modellierung und Prozessmessung im Sinn einer kontinuierlichen Selbstreflexion verändern den «Messgegenstand», sind also veränderungsrelevant. Zudem erfolgt eine Evaluation jeder einzelnen Psychotherapie, wobei sich die Evaluation nicht nur auf punktuelle Prä-Post-Erfassungen bezieht, sondern auf die Veränderung prozessualer Muster. So kann ein hochdynamisches Fluktuieren von Emotionen wie bei Borderline-Störungen oder dem rapid cycling von zyklothymen Störungen deutlich stabiler werden oder die eingeschränkte und rigide Emotionalität depressiver Patient"innen (emotional numbness) flexibler, adaptiver und komplexer, ohne dass sich dies im Mittelwert der Prozesse ausdrücken müsste.

Für die therapeutische Praxis ist es wichtig, die Fallkonzeption in Kooperation mit den Patient*innen an den Anfang zu stellen. Damit entsteht eine multiperspektivische und systemische Sicht auf das Problem und auf mögliche Ansatzpunkte für die Veränderung, und es entstehen die Variablen (Items eines individuellen Prozessfragebogens), mit denen der gesamte Therapieprozess gescannt wird. Danach, das heisst, sobald der Prozessfragebogen im SNS verfügbar ist, was wiederum in Kooperation mit den Patient*innen geschieht, startet das Monitoring, und darauf aufbauend der therapeutische Feedbackprozess. Demgegenüber würde ein Vorgehen, das lediglich datenbasierte korrelative Netzwerke von Symptomzeitreihen generiert (z. B. Borsboom \& Cramer, 2013), nur zu der Feststellung führen, dass Symptome Symptome produzieren, lässt Patient*innen aussen vor und setzt am falschen Ende des Prozesses an, nämlich dann, wenn bereits Zeitreihen vorliegen. Ein weiteres Problem von statistischen linearen Netzwerkanalysen (meist werden Varianten von Partialkorrelationen verwendet) besteht darin, dass sie nichtlineare Kausalrelationen (Systemzusammenhänge) auch in einfachen Systemen mit wenigen Variablen nicht identifizieren können. Aus Korrelationen lassen sich keine Kausalrelationen ableiten, vor allem keine nichtlinearen.

\section{Veränderungsmuster und Frühwarnindikatoren}

Im Verlauf von Psychotherapien depressiver Patient*innen sind bei hochfrequenten (d.h. täglichen) Prozesserfassungen spezifische Verlaufsmuster zu erkennen, die sich allerdings nach Stand unserer Kenntnis nicht grundlegend von den Prozessmustern bei anderen Diagnosen unterscheiden. Aufgrund der empirisch gut belegten Annahme, dass Psychotherapien selbstorganisierte Prozesse eines bio-psycho-sozialen Systems (nämlich des Patienten/der Patientin und seines/ihres sozialen Umfeldes) sind, kann man diskontinuierliche, also sprunghafte Veränderungen erwarten (vgl. Abb. 1), die dem entsprechen, was man in der Theorie als «Ordnungsübergänge» oder «Phasenübergänge» bezeichnet (Haken \& Schiepek, 2006). Diese spontanen Musterwechsel sind nicht notwendigerweise Reaktionen auf eine spezifische Intervention (vgl. die Fallbeispiele in Kronberger \& Aichhorn, 2015; Schiepek et al., 2009, 2016) und treten in erfolgreicheren Therapien deutlicher auf als in weniger erfolgreichen (Haken \& Schiepek, 2006; Helmich et al., 2020). Wie in einer Studie mit Zwangspatient*innen gezeigt werden konnte, haben diese Musterwechsel auch Entsprechungen in der Aktivierung neuronaler Strukturen, was bedeutet, dass psychische und neuronale Veränderungen als selbstorganisierte Musterwechsel synchron auftreten (Schiepek et al., 2013b). Man könnte also sagen, dass der Meso- und Makro-Outcome von Psychotherapien die Folge von selbstorganisierten Ordnungsübergängen ist.

In der Regel ereignen sich die Ordnungsübergänge nicht aus «heiterem Himmel», sondern werden durch bestimmte Veränderungen in der Dynamik eingeleitet. Man kann sich das so vorstellen, dass sich bestehende Muster - «Attraktoren» - auflösen und instabil werden, bevor sich ein neues Muster etabliert. Diese Destabilisierung drückt sich in sogenannten kritischen Instabilitäten aus, die kurz vor Musterübergängen auftreten und somit als Frühwarnindikatoren dienen können. Ein Mass für die kritische Instabilität von Zeitreihen ist die dynamische Komplexität, in der sich eine Veränderung der Amplitude, der Frequenz und der Streuung der Werte über die verfügbare Skala ausdrückt (Schiepek \& Strunk, 2010).

In einer Studie an 328 Patient*innen mit affektiven Störungen (einem Spektrum von rezidivierenden und chronischen Depressionen sowie zyklothymen Störungen) konnte eine Zunahme der dynamischen Komplexität im Sinne eines Frühwarnindikators vor einem Sprung in der Dynamik festgestellt werden (Olthof et al., 2019a). Die Patient"innen wurden stationär behandelt und ihre Verläufe mit dem Therapie-Prozessbogen täglich erfasst (die Daten stammen aus dem Grossdatensatz zur SNSbasierten Prozess- und Outcome-Forschung). Die Studie konzentrierte sich auf die Identifikation von sudden gains oder sudden losses, also sprunghafter Veränderungen des Niveaus einer Zeitreihe, in diesem Fall des Faktors «Symptom- und Problemintensität» des TPB. Die Abschnitte mit stabilem Niveau und deren sprunghaften Veränderungen wurden mit der Methode des recursive partitioning identifiziert (Abb. 3).

Die Studie konnte im Wesentlichen replizieren, was an einem kleineren Datensatz mit 94 Therapieverläufen unterschiedlicher Diagnosen festgestellt worden war, dass nämlich Ordnungsübergänge häufig von einer Zunahme kritischer Instabilität eingeleitet werden (Haken \& Schiepek, 2006). Dies ist ein Phänomen, das offenbar weder diagnose- noch psychotherapiespezifisch ist, denn es tritt auch bei Musterwechseln in der Motorik auf (Haken et al., 1985; Kelso et al., 1986) sowie in komplexen Systemen, die gar nichts mit dem Menschen zu tun haben, zum Beispiel in physikalischen Systemen wie dem Laser oder vor dem Übergang zu Konvektionsströmungen in Flüssigkeiten (Haken, 1990) oder auch vor dem Kippen von Ökosystemen (Scheffer et al., 2009). Therapierelevant sind die precursors (Frühwarnzeichen) insofern, als in Phasen der Destabilisierung ein anderes therapeutisches Vorgehen notwendig ist als in stabilen Phasen. So kommt es vor einem möglichen Musterwechsel darauf an, die Balance 
zwischen Stabilitätsbedingungen (generisches Prinzip 1) und Fluktuationsunterstützung (deviation amplifying feedback, generisches Prinzip 5) besonders im Blick zu behalten. In der Arbeit mit depressiven Patient* innen ist zu berücksichtigen, dass kritische Instabilitäten nicht nur vor entwicklungsförderlichen Ordnungsübergängen (z.B. sudden gains) auftreten, sondern auch vor suizidalen Krisen. Wenngleich nach Stand des Wissens eine Langfristvorhersage suizidaler Krisen unmöglich erscheint, so ist immerhin eine kurzfristige Vorhersage möglich (Fartacek et al., 2016). Das SNS erhält dabei die Funktion eines Frühwarnsystems.
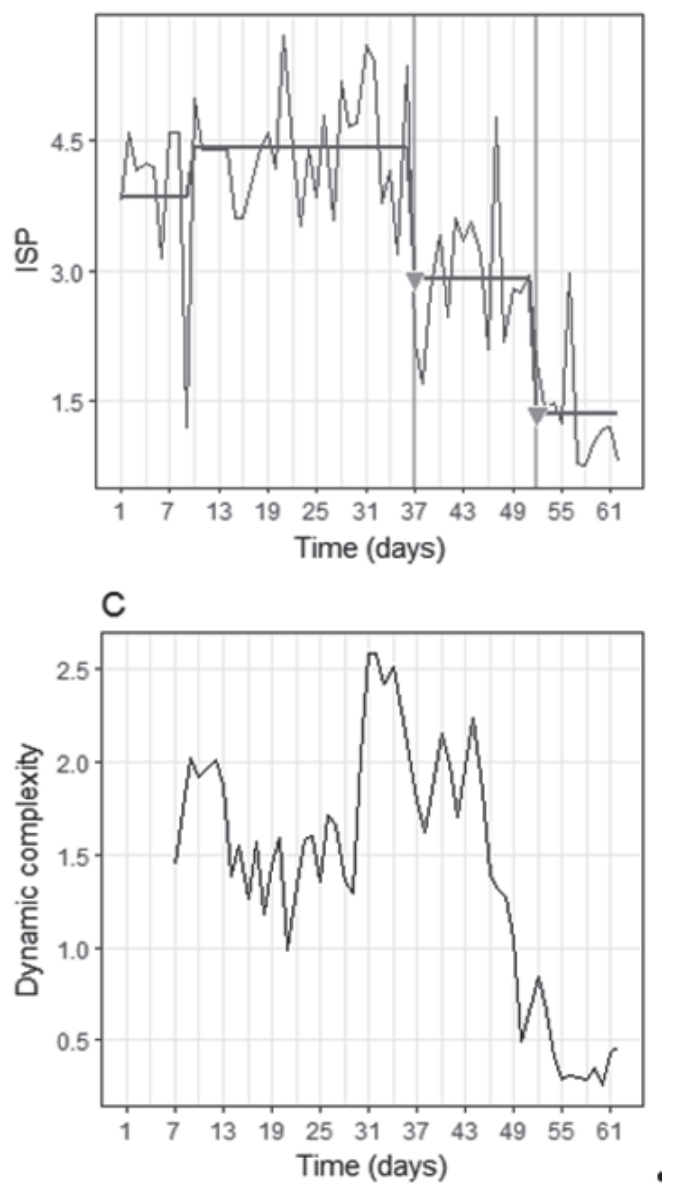

Abb. 3: Oben: Beispiel für die Partitionierung der Zeitreihe Symptom- und Problembelastung (hier ISP genannt) eines Patienten in stabile Segmente. Die Dreiecke markieren sogenannte sudden gains, also sprunghafte Reduzierungen der Symptombelastung. Unten: Die Entwicklung der dynamischen Komplexität dieser Zeitreihe, berechnet in einem überlagernden Gleitfenster von 7 Messpunkten (=Tagen) Breite (aus Olthof et al., 2019a).

Neben einer Zunahme kritischer Fluktuationen gibt es auch noch andere precursors oder Marker von sich anbahnenden Ordnungsübergängen. Einer ist die Zunahme der Synchronisation von Subsystemen oder von Elementen eines Systems, was in Psychotherapien bedeutet, dass sich Kognitionen (z. B. Selbstwahrnehmungen, Prozesseinschätzungen), Emotionen und Symptomintensitäten sehr kohärent verhalten. Im SNS drückt sich das in einer erhöhten Interkorrelation der Itemdynamik des verwendeten Prozessfragebogens aus (Haken \& Schiepek, 2006), wofür die Berechnung der Mittelwerte der absoluten (ohne Vorzeichen) Interitem-Korrelationen in einem laufenden Zeitfenster sowie eine ebenfalls dynamische Darstellung der Interkorrelationen in einer Matrix (Vorzeichen in Farbe dargestellt) zur Verfügung steht. Dieses Phänomen einer Synchronisationszunahme findet man übrigens auch in neuronalen Prozessen (Basar-Eroglu et al., 1996; Singer \& Gray, 1995 ) sowie in Ökosystemen am Rande eines ökologischen «Kippens» (Scheffer et al., 2009). Ein weiteres Merkmal speziell psychotherapeutischer Prozesse kurz vor einem Ordnungsübergang ist die Zunahme von Stress, emotionaler Belastung und Symptomen (Mahoney, 2001; Schiepek et al., 2016). Hayes und Strauss (1998) berichten über einen Zusammenhang zwischen Destabilisierung und emotionaler Intensität in Therapiesitzungen, was der Beobachtung vieler Praktiker*innen entspricht, dass es Patient*innen erst einmal schlechter geht, bevor eine Verbesserung eintritt.
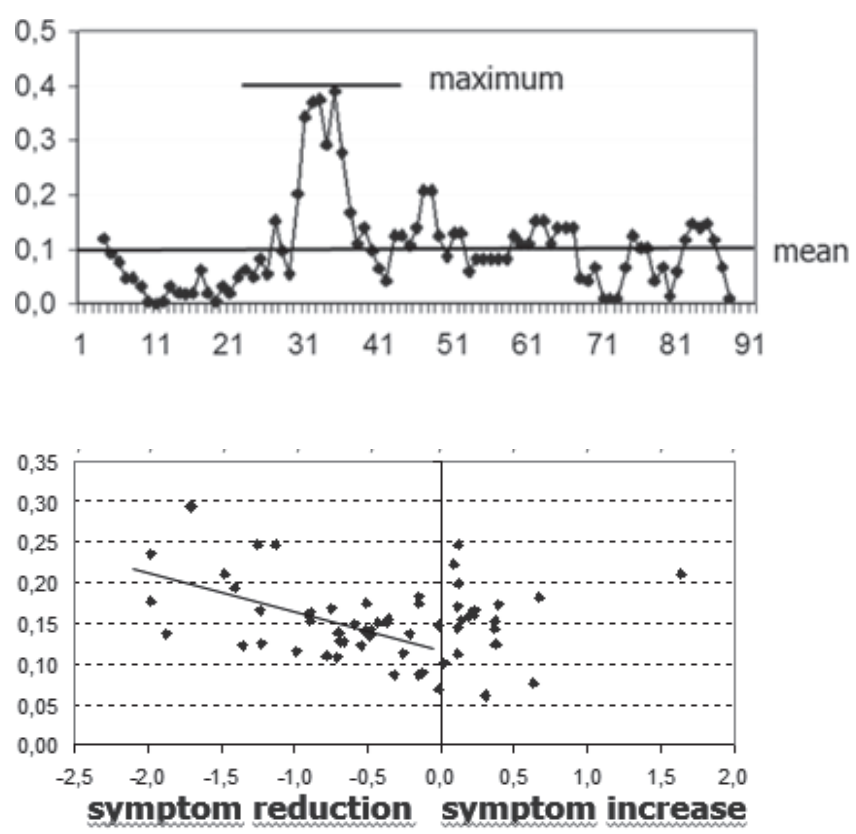

Abb. 4: Zusammenhang zwischen dem Peak der kritischen Instabilität und dem Therapieeffekt. Die Differenz zwischen dem Durchschnitt und dem Maximum der dynamischen Komplexität (oben) ist in der Grafik unten auf der y-Achse dargestellt. Die Problemreduktion ist auf der $x$-Achse ab der vertikalen Linie nach links dargestellt, die Problemzunahme ab der vertikalen Line nach rechts. Jeder Punkt repräsentiert eine $n$ Patientin/en. Die Korrelation zwischen der Höhe des Komplexitätspeaks und der Problemreduktion ist -.543 (p<.001) für die Patient*innen, die sich verbessert, und -.066 ( $p=.394)$ für die Patient*innen, die sich nicht verbessert oder verschlechtert haben. Der Effekt wurde gemessen mit einer Kombination aus dem Gießener Beschwerdebogen (GBB, Brähler \& Scheer, 1995), dem Inventar Interpersoneller Probleme (IIP, Horowitz et al., 1994) und der Hospital Anxiety and Depression Scale-dt. Version (HADS-D, Herrmann et al., 1995) (s. Haken \& Schiepek, 2006, S. 416-422). 
Ein interessanter Zusammenhang besteht zwischen dem Grad der Destabilisierung und dem Effekt von Psychotherapien. Der Grad der Instabilität vor einem Ordnungsübergang scheint mit dem Behandlungserfolg direkt in Zusammenhang zu stehen, was eine Folge von (1) Destabilisierung bestehender Muster zu (2) Musterwechsel (Ordnungsübergang) zu (3) Therapieeffekt nahelegt. Dieser Zusammenhang wurde in einer multidiagnostischen Patient*innengruppe (Haken \& Schiepek, 2006; Abb. 4), bei Zwangspatient*innen (Schiepek et al., 2014), bei Patient*innen mit Persönlichkeitsstörungen (Hayes \& Yasinski, 2015) und auch bei Adoleszent*innen (Lichtwarck-Aschoff et al., 2012) gefunden. Van de Leemput et al. (2014) berichten darüber mit Bezug auf Patient*innen mit affektiven Störungen (v. a. Depression) und unser Kooperationsteam mit der Radboud Universität Nijmegen (Olthof et al., 2019b) ebenfalls. Die Analyse beruht auf den Prozessdaten von 328 Patient*innen mit affektiven Störungen (v.a. einzelne Episoden, mehrfache Episoden und chronische Depression, vgl. die zuvor erwähnte Studie von Olthof et al., 2019a). Das Hautergebnis zeigte, dass bei den Patient*innen, die sich verbessert hatten, ein deutlicherer Zusammenhang zwischen der Intensität der dynamischen Komplexität und der Verbesserung (Symptomreduktion im ICD-10 basierten Symptomrating, Tritt et al., 2013) vorliegt als bei den Patient*innen, die sich nicht verbessert hatten (Abb. 5).

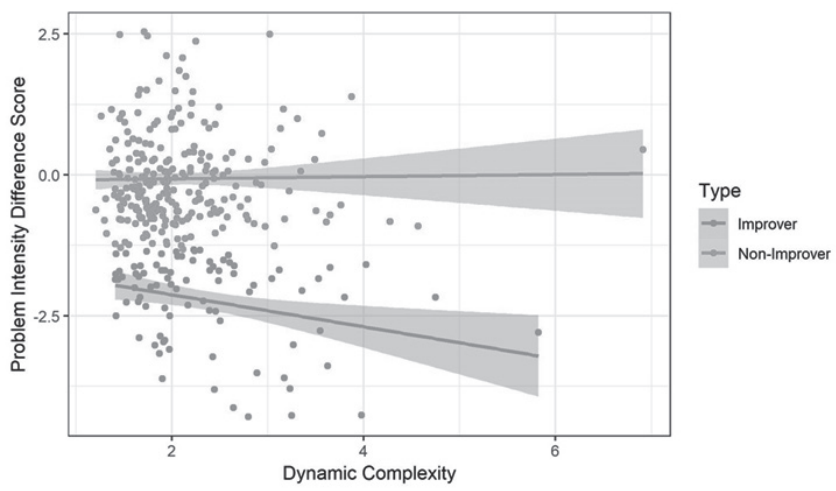

Abb. 5: Zusammenhang zwischen Problemreduktion bzw. Problemzunabme (y-Achse) und dynamischer Komplexität (x-Achse) bei Patient*innen, die sich verbessert hatten (untere Linie), und denen, die sich nicht verbessert hatten (obere Linie) (Quelle: Olthof et al., 2019b)

\section{Neuronale Aktivierungsmuster bei Depression}

Neben den psychologischen Korrelaten und Prozessmustern der Therapie von Depression gibt es zahlreiche, möglicherweise störungsspezifische biologische Marker und Befunde, zum Beispiel zu Stress, Traumatisierung und Depression (Depression als Stress-Folgeerkrankung), Herzinsuffizienz, endokrinen und immunologischen Korrelaten, Epigenetik, und auch zu neuronalen Mechanismen. Auf der Ebene neuronaler Aktivierungsmuster werden zum Beispiel eine Lateralitätshypothese im Sinne einer verscho- benen präfrontalen Aktivierung, eine Dysregulation des limbischen Systems (z. B. verstärkte Amygdala-Aktivierung), oder eine eingeschränkte Top-Down-Regulation von kortikalen auf limbische Strukturen diskutiert. Klar dürfte inzwischen sein, dass Depression nicht durch lokalisierte Über- oder Unteraktivierungen in einzelnen Hirnregionen erklärbar ist, sondern dass es sich eher um Netzwerke vieler Strukturen handelt, die dysreguliert funktionieren. In einer Meta-Analyse haben wir die Befundlage zur Neurokonnektivität bei Depression zusammengetragen (Studien aus den Jahren 2002-2015, die neuronale Auffälligkeiten bei depressiven im Vergleich zu gesunden Proband*innen verglichen; Helm et al., 2018), wobei vor allem eine Beteiligung des orbitofrontalen und -medialen Kortex, des anterioren cingulären Kortex, der Amygdalae, des Hippocampus, des Cerebellums und verschiedener Basalganglien-Strukturen deutlich wurde. Deutlich wurde aber auch die grosse Variabilität der Befunde in Abhängigkeit von der Art der Messung (z. B. strukturelles MRT, funktionelles MRT, EEG), der Art der Stimulation während der Messung (resting state oder verschiedene Arten von Stimulation oder durchzuführender Aufgaben) oder der Art der statistischen Vor- und Weiterverarbeitung der Rohdaten bzw. der Signale (BOLD oder EEG-Elektroden, z. B. als effektive oder funktionale Konnektivität; vgl. Abb. 6 und 7). Technisch gesprochen erschien die Methodenvarianz grösser als die Varianz des Phänomens, also der depressionsspezifischen neuronalen Netzwerkaktivität. Auch scheint es keine spezifische neuronale Über- oder Unterkonnektivität zwischen Hirnregionen zu geben, die charakteristisch für Depression sein könnte. Neben einer methodischen Vereinheitlichung von Versuchsdurchführungen und Stimulationen richtet sich eine weitere Schlussfolgerung auf die Notwendigkeit, die zeitliche Dynamik der Aktivierung von Netzwerken zu berücksichtigen. Das Gehirn ist ein hochdynamisches System, das auf der Meso-Ebene viele neuronale Strukturen einbezieht und selbstorganisiert permanent unterschiedliche Konnektivitätsmuster hervorbringt. Eine durch zeitliche Mittelung produzierte «durchschnittliche» Konnektivität bringt unser Verständnis depressionsspezifischer (wenn es das denn überhaupt gibt) neuronaler Dynamik nicht weiter.

Eine der weltweit führenden Arbeitsgruppen auf dem Gebiet der Dynamik neuronaler Netze ist die um Viktor Jirsa am Institut des Neurosciences des Systèmes am Institut National de la Santé et de la Recherche Médicale/ Université Aix en Provence/Marseille. Eine der dort entwickelten Methoden zur funktionellen Netzwerkdynamik (Hansen et al., 2015) konnten wir in einer Studie zur Veränderung der neuronalen Aktivierung im Laufe der Therapie von Patient*innen mit Zwangsstörungen und komorbider Depression anwenden (Schiepek et al., i. V.). Untersucht wurden 17 Patient*innenen und 17 gesunde Kontrollpersonen mit vier bis fünf fMRT-Scans im Laufe von drei bis vier Monaten stationärer Psychotherapie (Universitätsklinik für Psychiatrie, Psychotherapie und Psychosomatik, Salzburg). Neben täglichen Selbsteinschätzungen und der Erfassung zahlreicher biochemischer (endokriner und immunologischer) Parameter wurde in den Scans ein 

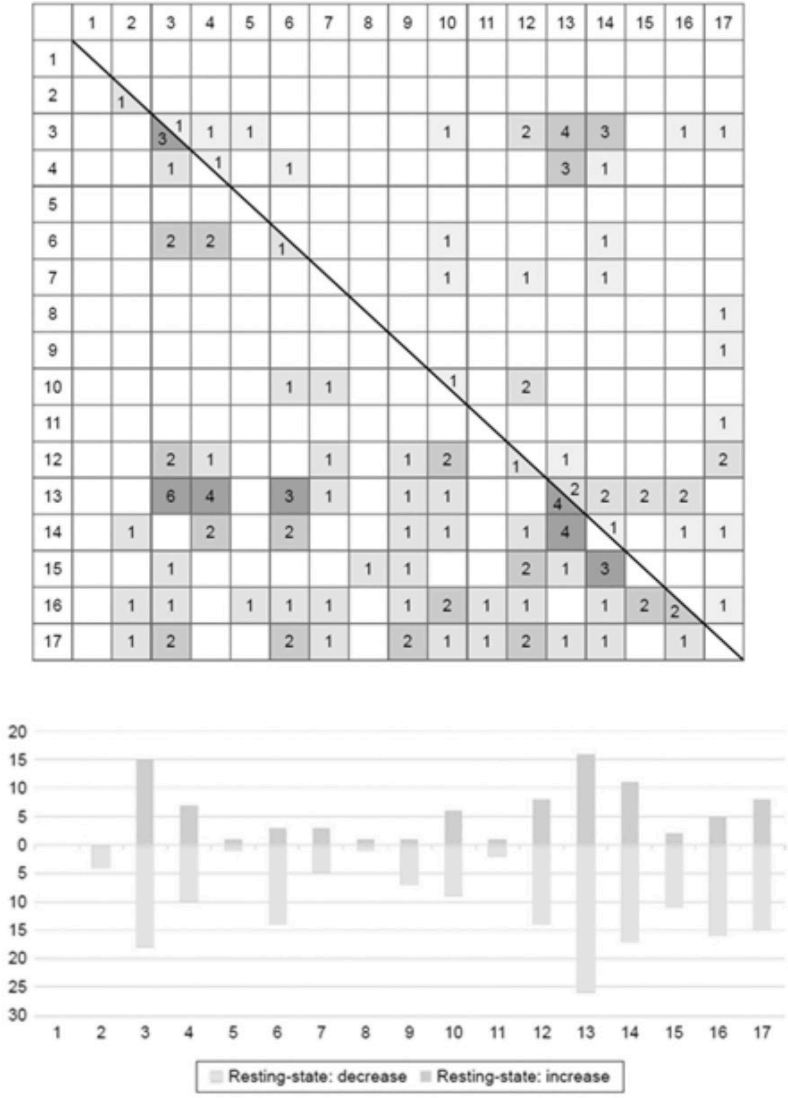

Abb. 6: Synopse der Befunde zur neuronalen Konnektivität depressiver Patient"innen (MDD) verglichen mit gesunden Kontrollpersonen, erfasst mit funktioneller Magnet-Resonanz-Tomographie (MRT) obne Stimulation (resting state). Die Matrix zeigt pathologisch veränderte Konnektivitäten zwischen 17 verschiedenen Hirnregionen, wobei die linke untere Dreiecksmatrix reduzierte Konnektivität und die rechte obere Dreiecksmatrix erhöhte Konnektivität anzeigt. Die Zablen in den Zellen beziehen sich auf die Anzahl der Studien, in denen die entsprechende veränderte Konnektivität gefunden wurde. Unten: Histogramm zur Anzahl der Studien, die in den 17 verschiedenen Hirnregionen eine veränderte Konnektivität mit mindestens einer anderen Region nachweisen konnten. Nach unten: reduzierte Konnektivität, nach oben: verstärkte Konnektivität. (1) Frontaler Pole, (2) orbitofrontaler Kortex, (3) lateraler frontaler Kortex, (4) medialer frontaler Kortex, (5) sensorimotorischer Kortex zentral, (6) superiorer Parietallappen, (7) inferiorer Parietallappen, (8) temporaler Pol, (9) superiorer Temporallappen, (10) mittlerer Temporallapen, (11) inferiorer Temporallapen, (12) medialer Temporallappen, (13) zingulärer Kortex, (14) Basalganglien/Thalamus, (15) Insula, (16) Occipitallappen, (17) Cerebellum.

umfassendes Stimulationsparadigma realisiert, wobei wir uns hier auf den Zeitabschnitt eines zehnminütigen resting state pro Scan beziehen. Die funktionelle Neurokonnektivität zwischen 119 neuroanatomischen Strukturen wurde

\begin{tabular}{|l|l|l|l|l|l|l|l|l|l|l|l|l|l|l|l|l|l|}
\hline & 1 & 2 & 3 & 4 & 5 & 6 & 7 & 8 & 9 & 10 & 11 & 12 & 13 & 14 & 15 & 16 & 17 \\
\hline 1 & & & & & & & & & & & & & & & & & \\
\hline 2 & & & & & & 1 & & & & 1 & & 1 & & 1 & 1 & 1 & 1 \\
\hline 3 & & & 1 & & & & & & & & & & 1 & & 1 & & \\
\hline 4 & & & & & & & & & & & & & & & & & \\
\hline 5 & & & & & & & & & & & & & & & 1 & & \\
\hline 6 & & & & & & & & & & & & & & & & & \\
\hline 7 & & & & & & & & & & & & & & & & & \\
\hline 8 & & & & & & & & & & & & & & & & & \\
\hline 9 & & & & & & & & & & & & & & & & & \\
\hline 10 & & & & & & & & & & & & 1 & & 1 & & \\
\hline 11 & & & & & & & & & & & & & & & & \\
\hline 12 & & 1 & & & & & & & & & 1 & 1 & 1 & 2 & & \\
\hline 13 & & & 2 & & & 1 & & & 1 & 1 & & 1 & 1 & 1 & & & \\
\hline 14 & & & 2 & 1 & 1 & & & & & & & & 1 & 1 & 1 & & \\
\hline 15 & & & 1 & & & & & & & & & & 1 & 1 & & 1 & 1 \\
\hline 16 & & & & & & & & & & & & & & & & & \\
\hline 17 & & & & & & & & & & & & & & & & & \\
\hline
\end{tabular}

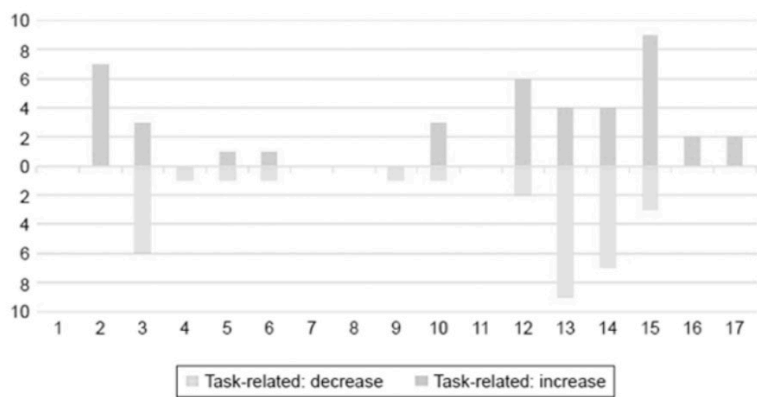

Abb. 7: Synopse der Befunde zur neuronalen Konnektivität depressiver Patient*innen (MDD) verglichen mit gesunden Kontrollpersonen, erfasst mit funktioneller Magnet-Resonanz-Tomographie (MRT) mit Stimulation bzw. im Scanner durchzufübrenden Aufgaben (vgl. ansonsten die Beschriftung zu Abb. 6).

in 49 nicht überlappenden Korrelationsmatrizen pro Scan erfasst, wobei unterschiedliche Fensterbreiten verwendet wurden (Abb. 8). Die Ergebnisse zeigen nicht nur deutliche Unterschiede der Korrelationsmuster zwischen den Hirnarealen (funktionelle Konnektivitätsdynamik), was eine dynamische Systembetrachtung des Gehirns nahelegt. Sie zeigen auch, dass die Veränderung der Konnektivitäten selbst eine relevante Information enthält, nämlich über die Flexibilität oder Rigidität der neuronalen Aktivität (Battaglia et al., 2020). Der Median der Korrelationswerte in den Zellen der Matrix kann sich von Matrix zu Matrix unterschiedlich stark verändern, was als jump length bezeichnet wird und angibt, wie schnell sich die neuronale Konnektivität in einem bestimmten Zeitraum (hier in der Periode des resting state) verändert. Korreliert man die Matrizen selbst untereinander, so entsteht eine Matrix der funktionellen Konnektivitätsdynamik (FCD), die eine Information über Ähnlichkeit oder Unähnlichkeit von Zeitabschnitten enthält (Zeit x Zeit-Matrix; Deco et al., 2013). Jump length und FCD können als Masse neuronaler Rigidität und Flexibilität interpretiert werden (Hansen et al., 2015; Battaglia et al., 2020). Unsere Befunde zeigen, 
dass die Sprunglänge in einem deutlichen (negativen) Zusammenhang mit Depressivität steht: höhere BDI-Werte (BDI-II: Beck Depression Inventory II; Hautzinger et al., 2009) korrespondieren mit niedrigeren Sprunglängen der neuronalen Konnektivität, mit einer Korrelation von -.474 ( $p=.007$; korrigiert mit False Discovery Rate: $p=.017$ ). Ein ähnlicher negativer Zusammenhang zwischen neuronaler Flexibilität und Psychopathologie liegt auch für andere klinische Beeinträchtigungen wie Angst und Stress (beide erhoben mit den Depressions-Angst-Stress-Skalen; Nilges \& Essau, 2015) sowie für die Zwangsskala des SCL-90 (Glöckner-Rist \& Stieglitz, 2012) vor. Die Befunde korrespondieren mit der Annahme reduzierter neuronaler, psychischer und physiologischer Flexibilität und Adaptivität als möglicher gemeinsamer Nenner von Psychopathologie (Haken \& Schiepek, 2006; Kashdan \& Rottenberg, 2010). In derselben Studie konnten wir feststellen, dass sich die die neuronale Flexibilität (Median der jump length) über die einzelnen fMRT-Scans und damit über die Therapie hinweg signifikant erhöht: Lag sie zu Beginn unter dem Konfidenzintervall des Durchschnitts der gesunden Kontrollpersonen, stieg sie zum Ende (letzter Scan) über dieses Konfidenzintervall hinaus an (Schiepek et al., i. V.).

Bei den zuvor dargestellten Befunden zur funktionellen Konnektivitätsdynamik stellt sich die Frage, ob eine neuromodulatorische Intervention in einem bestimmten Hirnareal ausreichend sein wird, um hochkomplexe Netzwerkdynamiken zu verändern. Die Methoden müssten möglicherweise dahin entwickelt werden, die Netzwerkdynamik relevanter neuronaler Systeme zu messen, zu analysieren und minimal zeitverzögert an unterschiedlichen Stellen (multi-site) zu stimulieren. Bis zur Entwicklung einer solchen multi-site-Feedback-Technologie der nichtinvasiven Neuromodulation ist sicher noch ein weiter Weg zurückzulegen. Auf alle Fälle müssten die Effekte auch von lokaler Neurostimulation auf die Netzwerkdynamik des Gehirns detailliert untersucht werden, zum Beispiel mit kombiniertem EEG-fMRT. Eine Kombination mit Psychotherapie scheint sinnvoll, nicht nur wegen der Befunde zu verbesserten tDCS-Effekten bei spezifisch kombinierten mentalen Aktivitäten, sondern auch, weil unser Gehirn Veränderungen (auch klinischen Verbesserungen) durch eigene Aktivität im Sinne einer Autorenschaft des Selbst deutlich von fremdinduzierten Effekten - sei es durch Medikation oder durch Neurostimulation - unterscheidet. Erfolge in eigener Autorenschaft ändern nicht nur Symptome, sondern auch unsere Selbstwahrnehmung, unsere Selbstwirksamkeit und unser Selbstwertgefühl.

\section{Schlussfolgerungen}

Es lassen sich in psychotherapeutischen Verläufen von Patient*innen mit Depression bestimmte Prozessmuster identifizieren. Ob diese Prozessmuster aber ausschliesslich bei Depression auftreten, ist nicht sicher. Nach Stand der Erkenntnis kommen sie auch in der Behandlung anderer Störungsbilder vor, sind also eher transdiagnostisch als depressionsspezifisch. Möglicherweise sind diskontinuier-

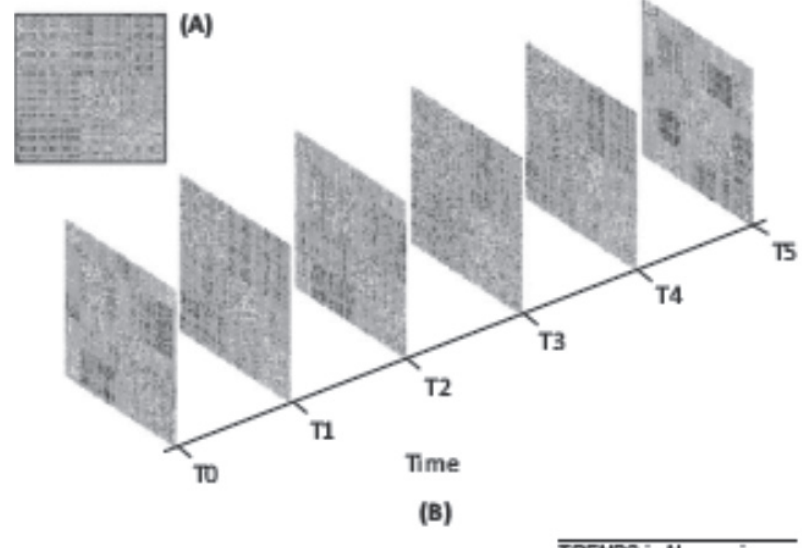

TREMOS in Neurosianoes

Abb. 8: Dynamik funktioneller Konnektivitätsmuster. (A) Beispiel für die funktionelle Konnektivität zwischen Hirnarealen, wobei die Konnektivtäten als Korrelationsstärken zwischen dem BOLD-Signal der Hirnareale in Graustufen dargestellt sind. (B) Abfolge von funktionellen Konnektivitätsmatrizen in aufeinanderfolgenden Zeitabschnitten (Quelle: Deco et al., 2013)

liche Ordnungsübergänge und ihre Frühwarnindikatoren sogar überhaupt nicht auf Psychotherapie beschränkt, sondern ein Merkmal der Selbstorganisation nichtlinearer komplexer Systeme generell. In Zukunft sollten wir transdiagnostische Cluster von Veränderungsmuster zu identifizieren versuchen, nicht zuletzt in Bezug auf erfolgreiche und weniger erfolgreiche Therapieverläufe.

Sowohl innovative Methoden der Fallkonzeption (z. B. idiografische Systemmodellierung) als auch ein darauf bezogenes Prozessmonitoring könnten die Veränderungsmotivation von Patient*innen als auch Therapieeffekte verbessern. Hierzu ist noch weitere Forschung sowohl zu den Wirkmechanismen als auch zu den therapeutischen Effekten notwendig. Auch diese Effekte sind wahrscheinlich nicht depressionsspezifisch. Neben einem standardisierten Prozessmonitoring kommt sinnvollerweise vor allem ein personalisiertes Monitoring infrage, mit Variablen, die in der qualitativen idiografischen Systemmodellierung erarbeitet wurden. Eine Netzwerkmodellierung nur von Symptomen (im Sinne von «Symptome produzieren Symptome») erweitert das Fallverständnis von Patient*innen nicht und bietet keine weiteren Perspektiven auf therapeutische Ansatzpunkte.

Eine Internet- und App-basierte Technologie des Prozessmonitorings, wie sie mit dem SNS vorliegt, bietet hierfür eine Schlüsseltechnologie. Datenerfassung (z.B. mit einer App), Generierung von Prozessfragebögen, Nutzung von Outcome-Fragebögen und Datenanalyse, vor allem nichtlineare Verfahren der Zeitreihenanalyse, einschliesslich einer umfassenden Visualisierung der Ergebnisse sind hierbei gleichzeitig möglich.

Psychotherapie kann mit nichtinvasiver Neurostimulation kombiniert werden, wobei bereits auch Psychotherapie allein als Methode der Neuromodulation verstanden werden kann. Die Effekte der Neurostimulation und der Psychotherapie auf die Dynamik neuronaler Netze müs- 
sen noch näher untersucht werden, einschliesslich einer geeigneten Stimulation als adaptive multi-site-FeedbackTechnologie (on demand).

\section{Literatur}

Bajbouj, M., Aust, S., Spies, J. et al. (2018). PsychotherapyPlus: augmentation of cognitive behavioral therapy (CBT) with prefrontal transcranial direct current stimulation (tDCS) in major depressive disorder - study design and methodology of a multicenter double-blind randomized placebo-controlled trial. European Archives of Psychiatry and Clinical Neuroscience, 268, 797-808.

Basar-Eroglu, C., Strüber, D., Kruse, P. et al. (1996). Frontal gamma-band enhancement during multistable visual perception. International Journal of Psychophysiology, 24, 113-125.

Battaglia, D., Boudou, T., Hansen, E.C.A. et al. (2020). Dynamic Functional Connectivity between Order and Randomness and its Evolution across the Human Adult Lifespan. NeuroImage, 117156. https://doi.org/10.1016/j.neuroimage.2020.117156

Borsboom, D. \& Cramer, A.O.J. (2013). Network analysis: An integrative approach to the structure of psychopathology. Annual Review of Clinical Psychology, 9, 91-121. https://doi.org/10.1146/ annurev-clinpsy-050212-185608

Brähler, E. \& Scheer, J.W. (1995). Der Gießener Beschwerdebogen $(G B B)$. Göttingen: Huber.

Brunoni, A.R., Valiengo, L., Baccaro, A. et al. (2013). The sertraline versus electrical current therapy for treating depression clinical study: results from a factorial, randomized, controlled trial. JAMA Psychiatry, 70, 383-391.

Butler, A.C., Chapman, J.E., Forman, E.M. \& Beck, A.T. (2006). The empirical status of cognitive-behavioral therapy: a review of meta-analyses. Clinical Psychology Review, 26, 17-31.

Deco, G., Jirsa, V.K. \& McIntosh, A.R. (2013). Resting brains never rest: computational insights into potential cognitive architectures. Trends in Neuroscience, 36, 268-274. https://doi.org/10.1016/j. tins.2013.03.001

DeRubeis, R.J., Siegle, G.J. \& Hollon, S.D. (2008) Cognitive therapy versus medication for depression: treatment outcomes and neural mechanisms. Nat Rev Neurosci, 9, 788-796.

Fartacek, C., Schiepek, G., Kunrath, S. et al. (2016). Real-time monitoring of nonlinear suicidal dynamics: methodology and a demonstrative case report. Frontiers in Psychology for Clinical Settings, 7: 130. https://doi.org/10.3389/fpsyg.2016.00130

Feeser, M., Prehn, K., Kazzer, P. et al. (2014). Transcranial direct current stimulation enhances cognitive control during emotion regulation. Brain Stimululation, 7, 105-112.

Glöckner-Rist, A. \& Stieglitz, R.-D. (2012). SCL-90-R - Die SymptomCheckliste von L.R. Derogatis (Deutsche Version). Psychologische Rundschau, 63, 73-75. https://doi.org/10.1026/0033-3042/a000103

Greenberg, P.E., Fournier, A.A., Sisitsky, T. et al. (2015). The economic burden of adults with major depressive disorder in the United States (2005 and 2010). J Clin Psychiatry, 76, 155-162.

Haken, H. (1990, 3. Aufl.). Synergetics. An Introduction. Nonequilibrium Phase Transitions in Physics, Chemistry, and Biology. Heidelberg: Springer.

Haken, H., Kelso, J.A.S. \& Bunz, H. (1985). A theoretical model of phase transition in human hand movements. Biological Cybernetics, 51, 347-356.

Haken, H. \& Schiepek, G. (2006, 2. Aufl. 2010). Synergetik in der Psychologie. Selbstorganisation verstehen und gestalten. Göttingen: Hogrefe.

Hansen, E.C.A., Battaglia, D., Spiegler, A. et al. (2015). Functional connectivity dynamics: Modeling the switching behavior of the resting state. NeuroImage, 105, 525-535. https://doi.org/10.1016/j. neuroimage.2014.11.001

Hautzinger, M., Keller, F. \& Kühner, C. (2009). BDI-II. Beck-Depressions-Inventar (Revidierte Fassung). Frankfurt/M.: Pearson Assessment.
Hayes, A.M. \& Strauss, J.L. (1998). Dynamic systems theory as a paradigm for the study of change in psychotherapy: an application to cognitive therapy for depression. Journal of Consulting and Clinical Psychology, 66, 939-947. http://doi.org/10.1037/0022-006X.66.6.939

Hayes, A.M. \& Yasinski, C. (2015). Pattern destabilization and emotional processing in cognitive therapy for personality disorders. Frontiers in Psychology, 6(FEB), 1-13. http://doi.org/10.3389/ fpsyg.2015.00107

Helm, K., Viol, K., Weiger, T.M. et al. (2018). Neuronal connectivity in Major Depressive Disorder: A systematic review. Neuropsychiatric Disease and Treatment, 14, 2715-2737. https://doi. org/10.2147/NDT.S170989

Helmich, M.A., Wichers, M., Olthof, M.J. et al. (2020). Sudden gains in day-to-day change: Revealing nonlinear patterns of individual improvement in depression. Journal of Consulting and Clinical Psychology, 88(2), 119-127. https://doi.org/10.1037/ccp0000469.

Herrmann, C., Buss, U. \& Snaith, R.P. (1995). HADS-D. Hospital Anxiety and Depression Scale - Deutsche Version. Bern: Huber.

Horowitz, L.M., Strauß, B. \& Kordy, H. (1994). Inventar zur Erfassung interpersonaler Probleme - Deutsche Version (IIP-D). Manual. Weinheim: Beltz.

Kashdan, T.B. \& Rottenberg, J. (2010) Psychological flexibility as a fundamental aspect of health. Clinical Psychology Review, 30, 865-878. https://doi.org/10.1016/j.cpr.2010.03.001

Kelso, J.A.S., Scholz, J.P. \& Schöner, G. (1986). Non-equilibrium phase transitions in coordinated biological motion: critical fluctuations. Physics Letters A, 118, 279-284.

Kronberger, H. \& Aichhorn, W. (2015). Selbstorganisation in der stationären Psychotherapie. Göttingen: Hogrefe.

Lichtwarck-Aschoff, A., Hasselman, F., Cox, R.F.A. et al. (2012). A characteristic destabilization profile in parent-child interactions associated with treatment efficacy for aggressive children. Nonlinear Dynamics, Psychology, and Life Sciences, 16, 353-379.

Nilges, P. \& Essau, C. (2015). Die Depressions-Angst-Stress-Skalen. Der Schmerz, 29, 649-657. https://doi.org/10.1007/s00482-015-0019-z

Mahoney, M.J. (1991). Human Change Processes. The Scientific Foundation of Psychotherapy. New York: Basic Books.

Meron, D., Hedger, N., Garner, M. \& Baldwin, D.S. (2015). Transcranial direct current stimulation (tDCS) in the treatment of depression: systematic review and meta-analysis of efficacy and tolerability. Neuroscience \& Biobehavioral Review, 57, 46-62.

Olchanski, N., McInnis Myers, M., Halseth, M. et al. (2013). The economic burden of treatment-resistant depression. Clin Ther, 35, 512-522.

Olthof, M., Hasselman, F., Strunk, G. et al. (2019a). Critical fluctuations as an early-warning signal for sudden gains and losses in patients receiving psychotherapy for mood disorders. Clinical Psychological Science. https://doi.org/10.1177/2167702619865969

Olthof, M., Hasselman, F., Strunk, G. et al. (2019b). Destabilization in self-ratings of the psychotherapeutic process is associated with better treatment outcome in patients with mood disorders. Psychotherapy Research. https://doi.org/10.1080/10503307.2019.1633484

Rufer, M. (2012). Erfasse komplex, handle einfach: Systemische Psychotherapie als Praxis der Selbstorganisation. Göttingen: Vandenhoeck \& Ruprecht.

Scheffer, M., Bascompte, J., Brock, W.A. et al. (2009). Early-warning signals for critical transitions. Nature Reviews, 461(3), 53-59. https://doi.org/10.1038/nature08227

Schiepek, G. (2019). Kein Klient ist «der Fall» von irgendwas - Das Spannungsfeld von individueller Fallkonzeption, Prozesssteuerung und Verallgemeinerung. In M. Rufer \& C. Flückiger (Hrsg.), Essentials der Psychotherapie (S. 83-95). Bern: Hogrefe.

Schiepek, G., Aichhorn, W. \& Schöller, H. (2018a). Monitoring change dynamics - a nonlinear approach to psychotherapy feedback. Chaos \& Complexity Letters, 11(3), 355-375.

Schiepek, G., Aichhorn, W., Schöller, H. \& Kronberger, H. (2018b). Prozessfeedback in der Psychotherapie. Methodik, Visualisierung und Fallbeispiel. Psychotherapeut, 63(4), 306-314. https://doi. org/10.1007/s00278-018-0272-6 
Schiepek, G., Eckert, H. \& Kravanja, B. (2013a). Grundlagen systemischer Therapie und Beratung. Göttingen: Hogrefe.

Schiepek, G., Gelo, O., Viol, K. et al. (2020). Complex individual pathways or standard tracks? A data-based discussion on the trajectories of change in psychotherapy. Counselling \& Psychotherapy Research. https://doi.org/10.1002/capr.12300

Schiepek, G., Stöger-Schmidinger, B., Aichhorn, W. et al. (2016). Systemic case formulation, individualized process monitoring, and state dynamics in a case of dissociative identity disorder. Frontiers in Psychology for Clinical Settings, 7, 1545. https://doi.org/10.3389/ fpsyg.2016.01545

Schiepek, G., Stöger-Schmidinger, B., Kronberger, H. et al. (2019). The Therapy Process Questionnaire. Factor analysis and psychometric properties of a multidimensional self-rating scale for high-frequency monitoring of psychotherapeutic processes. Clinical Psychology \& Psychotherapy, 26, 586-602. https://doi.org/10.1002/cpp.2384

Schiepek, G. \& Strunk, G. (2010). The identification of critical fluctuations and phase transitions in short term and coarse-grained time series - a method for the real-time monitoring of human change processes. Biological Cybernetics, 102(3), 197-207. https://doi. org/10.1007/s00422-009-0362-1

Schiepek, G., Tominschek, I., Heinzel, S. et al. (2013b). Discontinuous patterns of brain activation in the psychotherapy process of obsessive compulsive disorder: converging results from repeated fMRI and daily self-reports. PloS ONE, 8(8), e71863.

Schiepek, G., Tominschek, I. \& Heinzel, S. (2014). Self-organization in psychotherapy - testing the synergetic model of change processes. Frontiers in Psychology for Clinical Settings, 5, 1-11. https://doi.org/10.339/fpsyg.2014.01089

Schiepek, G., Tominschek, I., Karch, S. et al. (2009). A controlled single case study with repeated fMRI measures during the treatment of a patient with obsessive-compulsive disorder: Testing the nonlinear dynamics approach to psychotherapy. The World Journal of Biological Psychiatry, 10(5), 658-668. https://doi. org/10.1080/15622970802311829

Schiepek, G., Viol, K., Aas, B. et al. (i. V.). Pathologically reduced neural flexibility improves during psychotherapy of OCD patients.

Segrave, R.A., Arnold, S., Hoy, K. \& Fitzgerald, P.B. (2014). Concurrent cognitive control training augments the antidepressant efficacy of tDCS: a pilot study. Brain Stimulation, 7, 325-331.

Shedler, J. (2018). Where is the evidence for «evidence-based therapy»? Psychiatric Clinics of North America, 41(2), 319-329. https://doi.org/10.1016/j.psc.2018.02.001

Singer, W. \& Gray, C.M. (1995). Visual feature integration and the temporal correlation hypothesis. Ann. Rev. Neurosci., 18, 555-586.

Tritt, K., von Heymann, F., Zaudig, M. et al. (2013). The ICD-10Symptom-Rating (ISR): Brief Description. Munich, Germany: Institute for Quality Development in Psychotherapy and Psychosomatics.

van de Leemput, I.A., Wichers, M., Cramer, A.O.J. et al. (2014). Critical slowing down as early warning for the onset and termination of depression. Proceedings of the National Academy of Sciences USA, 111, 87-92. http://doi.org/10.1073/pnas.1312114110

Wampold, B.E., Flückiger, C., del Re, A.C. et al. (2017). In pursuit of truth: A critical examination of meta-analyses of cognitive behavior therapy. Psychotherapy Research, 27, 14-32. https://doi.org/10 .1080/10503307.2016.1249433

Wampold, B.E, Imel, Z.E. \& Flückiger, C. (2018). Die Psychotherapie Debatte. Göttingen: Hogrefe.

Whiteford, H.A., Degenhardt, L., Rehm, J. et al. (2013). Global burden of disease attributable to mental and substance use disorders: findings from the Global Burden of Disease Study 2010. Lancet, 382, 1575-1586.

Wolkenstein, L. \& Plewnia, C. (2013) Amelioration of cognitive control in depression by transcranial direct current stimulation. Biological Psychiatry, 73, 646-651.

Wolkenstein, L. \& Plewnia, C. (2013) Amelioration of cognitive control in depression by transcranial direct current stimulation. Biological Psychiatry, 73, 646-651.

\section{Depression - a Complex Dynamic System}

Abstract: This article focuses on some new developments in the treatment of Major Depressive Disorder with the question of how to optimize psychotherapeutic procedures. Following the intention of the editor of this journal, a short synopsis is given on the scientific and practical developments at the Institute of Synergetics and Psychotherapy Research at the Paracelsus Medical University Salzburg. One focus is on the personalization of psychotherapy, which concerns new methods of case formulation (e.g., Idiographic System Modelling and computer-based tools for its visualization) and technologies for high-frequency process-monitoring and -feedback. For this reason, we developed and validated the Synergetic Navigation System (SNS) which is an Internet- and App-based application for data collection, data analysis, and visualization of change dynamics and outcome. Based on this methodology, it became evident that the psychotherapeutic processes of depressive disorders are characterized by discontinuous patterns, so called «order transitions». The precursors of these discontinuous transitions are critical fluctuations represented by increased dynamic complexity, and an increase in the synchronization of the inter-item dynamics of the therapy process questionnaire. The amount of critical instabilities occurring before order transitions revealed to be predictive for treatment outcome. Beside clinical improvement also neural connectivity patterns are changing by psychotherapy, as could be shown in repeated fMRI scans during inpatient treatment of comorbid depressive patients with obsessive compulsive disorder. The functional connectivity dynamics of 119 brain regions was assessed during the resting state periods of the repeated scans, showing that neural rigidity (a loss of flexibility and fluidity) is associated with psychopathology (e.g., depression, BDI), but flexibility increases during psychotherapy. Finally, the option of combining psychotherapy with noninvasive neurostimulation will be discussed.

Key words: personalized psychotherapy, process monitoring, therapeutic patterns of change, order transitions, precursors and early warnings, neural connectivity patterns, noninvasive neurostimulation

\section{Depressione - un sistema dinamico complesso}

Riassunto: il presente studio si focalizza sulle ultime scoperte della ricerca sulla depressione, con particolare riguardo al trattamento di tale patologia in ambito psicoterapeutico. Su richiesta della redazione, verranno passati in rassegna anche gli sviluppi scientifici e pratici registrati presso l'Institut für Synergetik und Psychotherapieforschung (Istituto di sinergetica e ricerca psicoterapeutica) della Paracelsus Medizinischen Privatuniversität (Università medica privata Paracelsus) di Salisburgo e dei gruppi di ricercatori che vi collaborano. Uno dei temi principali è rappresentato dalla personalizzazione della psicoterapia e riguarda sia i più recenti metodi di concettualizzazione del caso del paziente (ad es. modellazione sistemica idiografica e relativa visualizzazione computerizzata) sia la metodologia di monitoraggio (ad alta frequenza) e il feedback dei processi neuronali che intervengono durante la terapia. A questo riguardo è stato sviluppato e testato un sistema di navigazione sinergetico basato su app e internet (SNS). Lo svolgimento delle sedute di psicoterapia di pazienti con depressione ha mostrato cambiamenti discontinui dei modelli dinamici (i cosiddetti passaggi 
ad altro stato di ordine), che sono generati da un'instabilità critica elevata (complessità dinamica) e da una sincronizzazione limitata nel tempo degli elementi dello schema del processo terapeutico. Un'elevata instabilità critica è inoltre predittiva di migliori risultati nel corso della terapia. In uno studio svolto su pazienti depressi e inoltre affetti da disturbi compulsivi, oltre a miglioramenti clinici, si sono evidenziati anche cambiamenti nella dinamica di connettività delle reti neuronali. Ripetuti esami effettuati tramite $\mathrm{fRMN}$ hanno evidenziato l'associazione negativa della flessibilità e della fluidità delle dinamiche neuronali con la psicopatologia (ad es. intensità della depressione, $\mathrm{BDI})$, tuttavia con un loro aumento nel corso della psicoterapia. Infine segue una valutazione delle possibilità di combinare la neurostimolazione non invasiva alla psicoterapia.

Parole chiave: terapia personalizzata, monitoraggio processi, modello dello svolgimento terapeutico, cambiamenti di ordine, indicatori precoci, modelli del processo neuronale, neurostimolazione non invasiva

\section{Der Autor}

Günter Schiepek, Univ.-Prof. Dr. phil. Dr. phil. habil, ist Leiter des Instituts für Synergetik und Psychotherapieforschung an der Paracelsus Medizinischen Privatuniversität Salzburg (PMU), Professor an der PMU sowie an der Ludwig-Maximilians-Universität München, Gastprofessor an der Sapienza Universität Rom, Geschäftsführer des Center for Complex Systems (Stuttgart/Salzburg), Mitglied der Europäischen Akademie der Wissenschaften und Künste (Academia Scientiarium et Artium Europaea) und Ehrenmitglied der Systemischen Gesellschaft (Deutscher Dachverband für systemische Forschung, Therapie, Supervision und Beratung).

\section{Kontakt}

Prof. Dr. Günter Schiepek

Institut für Synergetik und Psychotherapieforschung

Universitätsklinik für Psychiatrie, Psychotherapie und Psychosomatik

Paracelsus Medizinische Privatuniversität Ignaz Harrer Str. 79

A-5020 Salzburg

E-Mail: guenter.schiepek@ccsys.de 